\title{
BUILDING CONSTRUCTION LABOUR PRODUCTIVITY IN ARID CLIMATE ENVIRONMENT
}

\author{
BHATTI Imtiaz Ali ${ }^{\text {a,*, }}$, ABDULLAH Abd Halid ${ }^{\text {a }, \text { NAGAPAN Sasitharan }}$ a, \\ BHATTI Nabi Bux ${ }^{\text {b }}$, LAKHIAR Muhammad Tahir ${ }^{c}$, MAHAR Muntazir Mahdi ${ }^{\text {a }}$ \\ ${ }^{a}$ Universiti Tun Hussein Onn Malaysia Parit Raja, Faculty of Civil and Environmental Engineering, Johor, Malaysia, \\ e-mail:*Engrimtiaz290@gmail.com \\ ${ }^{\mathrm{b}}$ Mehran University of Engineering \& Technology, U.S.-Pakistan Centre for Advanced Studies in Water, Sindh, Pakistan \\ ${ }^{c}$ National University of Sciences and Technology Quetta Campus, Department of Civil Engineering, Balouchistan, Pakistan
}

Received: 24.09.2019 / Accepted: 05.10.2019/ Revised: 01.11.2019 / Available online: 10.12.2019

DOI: 10.2478/jaes-2019-0017

KEY WORDS: Construction, Production, Cost of Labour, building project, weather conditions, target quantity.

\begin{abstract}
:
Productivity is a significant aspect of construction industry that plays vital role for success and failure of any construction project. This industry generates $11 \%$ to $13 \%$ of GDP all around the globe and the cost of labour in any building project is $20 \%$ to $35 \%$ of the cost of Building. On daily basis labour utilizes $30 \%$ of time on productive activities rest $70 \%$ of the time is ruined in non-productive activities, there are multi factors which are affecting the labour production in construction industry hence this study provides an overview of productivity, Total Factor productivity, method used to measure accurate productivity in construction projects. The objective of this study is find out percentage up to what extent labour production is affected due to weather conditions, however this study is carried out in arid climate region in Month of June 2018, where minimum temperature was recorded 26.0 Celsius degree at 7:30 AM and Maximum was 47.80 Celsius degree at 3:00 PM. A descriptive survey research design approach was adopted using continuous observation method of study. Project work study manual served as the research instrument to collect the data on selected building sites for 30 working days. Data collected were analyzed using descriptive statics. The results show that average monthly production of mason gang was recorded with less production of $28.759 \%$, Carpentry gang with average monthly loss of production $16.74 \%$ \& steel fixer gang had average monthly loss of production was 12.188 . This concludes that prior to signing the contract for construction project. The location, environment, topography of region, capacity of construction operatives must be kept in mind to decide the proper timeline for the successful of project.
\end{abstract}

\section{INTRODUCTION}

Construction industry all around the world plays a dynamic role for the prosperity of any country (S. Sohu et al. 2018). This is a versatile industry which generates around 11 to $13 \%$ of GDP annually (I.A Bhatti et al., 2018). The cost of labour in any building construction project is $20 \%$ to $35 \%$ of the cost of Building (Buchan R. D., Fleming F. W., Kelly J. R., 1993). The time utilized by labour on daily basis on productive activities is average about $30 \%$ of total time available for construction work. The rest of the time about $70 \%$ is wasted on nonproductive activities, delays and added activities (I.A Bhatti et al. 2018). Construction projects are unique very few projects are repeated, and experience gained on one site is not necessarily of benefit or directly applicable on other site (Mahamid, I. 2013). Projects locations, site topography, labor force vary from one project to another. Meanwhile this industry faces many challenges like time overrun, cost overrun, construction waste generation and management, health and safety challenges are the key challenges faced by this industry; however there is one predominant challenge of labor production which is the key concern for this industry (Oladiran, O. J. and Onatayo, D. 2019). labour production is severe concern which gives birth to time overrun and cost overrun problems within the construction projects (Gerek, İ. H., Erdis, E., Mistikoglu, G. \& Usmen, M. 2015). Construction productivity is affected because of multiple factors and different causes. The intensity of these factors and causes varies from site to site and from day to day (Jarkas, A. M. \& Bitar, C. G. 2012). The effect of these factors is to change the productivity. The Aim of this study is to determine the extent to which variability in labour productivity can be explained by variations in arid climate environment. To achieve this aim, it will be essential to develop consistent techniques for measuring productivity and factors which are

\footnotetext{
* Corresponding author: BHATTI Imtiaz Ali, e-mail: Engrimtiaz290@gmail.com
} 
affecting it. This study is conducted primarily in Middle East arid climate region.

\section{LITERATURE REVIEW}

Every construction project is based on Iron triangle constraint approach which has three basic elements; time, cost and quality. These three constraints are firmly connected together. Labour production is key constraint among these three elements and has direct interrelationship with triple constraints of iron triangle.

2.1 Definition of Productivity: when study was carried out in depth, it was found that there is no perfect definition for the word construction productivity. Even when definitions are consistent, different approaches to measuring input and output vary so greatly that a valid comparison between projects is almost impossible. Various definitions of the productivity have been encountered, these include the following
a. Efficiency
b. Effectiveness
c. Performance
d. Production

The general consent is to define the productivity as the ratio of output to input. In view of this, two approaches to productivity measurement emerges total factor productivity where all inputs and outputs are considered and single factor productivity where single factor is taken into consideration (Revianty Nurmeyliandari Nurhendi, Muhamad Azry Khoiry, Noraini Hamzah (2019).
2.2 Labour Productivity Measurement: There are numerous techniques involved in measuring the labour productivity especially in construction industry. Measurement of labour production is varies according to the nature of Project. Broadly, productivity measures can be classified as single factor productivity measures (relating a measure of output to a single measure of input) or multifactor productivity measures (relating a measure of output to a bundle of inputs). Another distinction, of relevance at the industry or firm level is between productivity measures that relate some measure of gross output to one or several inputs and those which use a value-added concept to capture movements of output (Kazaz, A., Ulubeyli, S., Acikara, T. \& Er, B. 2016).

\section{METHODOLOGY}

A descriptive research methodology approach was adopted using continuous observation method of study. Project work study manual served as the research instrument to collect the data on selected building site for 30 working days. On every working day temperature was recorded twice a day as shown in Table 1. First early in the morning and second it was recorded at mid noon time. Meanwhile the labour production was also going to be recorded simultaneously every day with respect to activity carried out by construction operatives at site as shown in Table $2,3 \& 4$

Table 1. Daily on-site Temperature Record

\begin{tabular}{|c|c|c|c|c|c|}
\hline \multicolumn{3}{|c|}{ Morning time } & \multicolumn{3}{|c|}{ After Noon Time } \\
\hline Date & Time & Temperature & Date & Time & Temperature \\
\hline $1-6-2018$ & \multicolumn{2}{|c|}{ Friday } & $1-6-2018$ & \multicolumn{2}{|c|}{ Friday } \\
\hline $2-6-2018$ & 09:00 AM & $29.0^{\circ} \mathrm{C}$ & $2-6-2018$ & 02:00 PM & $43.2^{\circ} \mathrm{C}$ \\
\hline $3-6-2018$ & 09:30 AM & $29.3^{\circ} \mathrm{C}$ & $3-6-2018$ & 01:45 PM & $42.8^{\circ} \mathrm{C}$ \\
\hline $4-6-2018$ & 10:00 AM & $32.4^{\circ} \mathrm{C}$ & $4-6-2018$ & 01:00 PM & $41.2^{\circ} \mathrm{C}$ \\
\hline $5-6-2018$ & 08:00 AM & $28.0^{\circ} \mathrm{C}$ & $5-6-2018$ & $02: 45 \mathrm{PM}$ & $42.0^{\circ} \mathrm{C}$ \\
\hline $6-6-2018$ & 07:30 AM & $26.4^{0} \mathrm{C}$ & 6-6-2018 & 03:00 PM & $39.6^{0} \mathrm{C}$ \\
\hline $7-6-2018$ & 07:45 AM & $26.8^{\circ} \mathrm{C}$ & $7-6-2018$ & 03:45 PM & $38.3^{\circ} \mathrm{C}$ \\
\hline $8-6-2018$ & \multicolumn{2}{|c|}{ Friday } & $8-6-2018$ & \multicolumn{2}{|c|}{ Friday } \\
\hline 9-6-2018 & 07:45 AM & $27.1{ }^{0} \mathrm{C}$ & 9-6-2018 & 01:15 PM & $42.7^{0} \mathrm{C}$ \\
\hline $10-6-2018$ & $07: 30 \mathrm{AM}$ & $27.9^{\circ} \mathrm{C}$ & $10-6-2018$ & 01:45 PM & $43.0^{\circ} \mathrm{C}$ \\
\hline $11-6-2018$ & 08:00 AM & $28.3^{0} \mathrm{C}$ & $11-6-2018$ & 02:00 PM & $41.8^{\circ} \mathrm{C}$ \\
\hline $12-6-2018$ & 09:00 AM & $31.1{ }^{\circ} \mathrm{C}$ & $12-6-2018$ & 03:00 PM & $41.3^{\circ} \mathrm{C}$ \\
\hline $13-6-2018$ & 09:30 AM & $32.0^{\circ} \mathrm{C}$ & 13-6-2018 & 03:45 PM & $40.5^{\circ} \mathrm{C}$ \\
\hline 14-6-2018 & 10:00 AM & $33.2{ }^{0} \mathrm{C}$ & 14-6-2018 & 03:15 PM & $40.9^{0} \mathrm{C}$ \\
\hline $15-6-2018$ & \multicolumn{2}{|c|}{ Friday } & $15-6-2018$ & \multicolumn{2}{|c|}{ Friday } \\
\hline $16-6-2018$ & 09:00 AM & $32.0^{\circ} \mathrm{C}$ & $16-6-2018$ & 02:00 PM & $39.8^{\circ} \mathrm{C}$ \\
\hline $17-6-2018$ & 09:30 AM & $32.3{ }^{0} \mathrm{C}$ & $17-6-2018$ & 01:45 PM & $40.1^{0} \mathrm{C}$ \\
\hline $18-6-2018$ & 10:00 AM & $33.0^{\circ} \mathrm{C}$ & $18-6-2018$ & 01:00 PM & $40.7^{0} \mathrm{C}$ \\
\hline 19-6-2018 & 08:00 AM & $30.2^{\circ} \mathrm{C}$ & 19-6-2018 & $02: 45 \mathrm{PM}$ & $41.2^{\circ} \mathrm{C}$ \\
\hline $20-6-2018$ & 07:30 AM & $29.8^{\circ} \mathrm{C}$ & $20-6-2018$ & 03:00 PM & $40.9^{\circ} \mathrm{C}$ \\
\hline $21-6-2018$ & 07:45 AM & $31.0^{\circ} \mathrm{C}$ & $21-6-2018$ & 03:45 PM & $40.3^{\circ} \mathrm{C}$ \\
\hline $22-6-2018$ & \multicolumn{2}{|c|}{ Friday } & $22-6-2018$ & \multicolumn{2}{|c|}{ Friday } \\
\hline 23-6-2018 & 07:45 AM & $30.3^{0} \mathrm{C}$ & 23-6-2018 & $01: 15 \mathrm{PM}$ & $42.3^{0} \mathrm{C}$ \\
\hline $24-6-2018$ & 07:30 AM & $30.1^{0} \mathrm{C}$ & $24-6-2018$ & $01: 45 \mathrm{PM}$ & $42.8^{0} \mathrm{C}$ \\
\hline $25-6-2018$ & 08:00 AM & $31.0^{\circ} \mathrm{C}$ & $25-6-2018$ & 02:00 PM & $43.0^{\circ} \mathrm{C}$ \\
\hline 26-6-2018 & 09:00 AM & $34.5{ }^{0} \mathrm{C}$ & $26-6-2018$ & 03:00 PM & $47.8^{0} \mathrm{C}$ \\
\hline $27-6-2018$ & 09:30 AM & $33.7^{0} \mathrm{C}$ & 27-6-2018 & 03:45 PM & $40.2^{0} \mathrm{C}$ \\
\hline $28-6-2018$ & 10:00 AM & $34.8^{\circ} \mathrm{C}$ & $28-6-2018$ & 03:15 PM & $40.7^{\circ} \mathrm{C}$ \\
\hline $29-6-2018$ & \multicolumn{2}{|c|}{ Friday } & $29-6-2018$ & \multicolumn{2}{|c|}{ Friday } \\
\hline $30-6-2018$ & 07:45 AM & $31.2{ }^{0} \mathrm{C}$ & $30-6-2018$ & $02: 45 \mathrm{PM}$ & $41.2^{\circ} \mathrm{C}$ \\
\hline
\end{tabular}




\subsection{Labour Production Record on Daily Basis}

3.1.1. Mason Gang Production: Mason gang was consisting of one charge hand, five masons and five helpers total ten numbers of operatives were involved in one gang. According to gang and masonry activities targets were already set by the company, there productivity was recorded on daily basis, however only block work activity was in progress on the construction site which was recorded on daily basis as shown in Table 2 .

Table 2. Production Record of Mason Gang

\begin{tabular}{|c|c|c|c|}
\hline Date & Production by Gang & Targeted Production & Remarks \\
\hline $1-6-2018$ & \multicolumn{3}{|c|}{ Friday } \\
\hline $2-6-2018$ & 486 & \multirow{6}{*}{$\begin{array}{l}700 \text { No of hollow Blocks of } 10 \mathrm{~cm} \text { in } \\
\text { size per day }(700 \times 6=4200) \text { per week. }\end{array}$} & \multirow{7}{*}{$26.071 \%$ less production than the targeted } \\
\hline 3-6-2018 & 510 & & \\
\hline $4-6-2018$ & 526 & & \\
\hline $5-6-2018$ & 555 & & \\
\hline $6-6-2018$ & 492 & & \\
\hline \multirow[t]{2}{*}{$7-6-2018$} & 536 & & \\
\hline & 3105 & 4200 & \\
\hline $8-6-2018$ & \multicolumn{3}{|c|}{ Friday } \\
\hline $9-6-2018$ & 370 & \multirow{6}{*}{$\begin{array}{l}500 \text { No of Solid Blocks of } 10 \mathrm{~cm} \text { in } \\
\text { size per day }(500 \times 6=2880) \text { per Week }\end{array}$} & \multirow{6}{*}{$22.966 \%$ less production than the targeted } \\
\hline $10-6-2018$ & 385 & & \\
\hline $11-6-2018$ & 428 & & \\
\hline $12-6-2018$ & 325 & & \\
\hline $13-6-2018$ & 348 & & \\
\hline \multirow[t]{2}{*}{$14-6-2018$} & 455 & & \\
\hline & 2311 & 3000 & \\
\hline 15-6-2018 & \multicolumn{3}{|c|}{ Friday } \\
\hline $16-6-2018$ & 492 & \multirow{6}{*}{$\begin{array}{l}750 \text { No of hollow blocks of } 20 \mathrm{~cm} \text { in } \\
\text { size per day }(750 \times 6=4500) \text { per Week }\end{array}$} & \multirow{6}{*}{$30.008 \%$ less production than the targeted } \\
\hline $17-6-2018$ & 512 & & \\
\hline $18-6-2018$ & 545 & & \\
\hline $19-6-2018$ & 586 & & \\
\hline $20-6-2018$ & 499 & & \\
\hline \multirow[t]{2}{*}{ 21-6-2018 } & 512 & & \\
\hline & 3146 & 4500 & \\
\hline $22-6-2018$ & \multicolumn{3}{|c|}{ Friday } \\
\hline $23-6-2018$ & 223 & \multirow{6}{*}{$\begin{array}{l}400 \text { No of solid blocks of } 20 \mathrm{~cm} \text { in size } \\
\text { per day }(400 \times 6=2400) \text { per week }\end{array}$} & \multirow{6}{*}{$35.291 \%$ less production than the targeted } \\
\hline $24-6-2018$ & 241 & & \\
\hline $25-6-2018$ & 275 & & \\
\hline $26-6-2018$ & 258 & & \\
\hline $27-6-2018$ & 275 & & \\
\hline \multirow[t]{2}{*}{$28-6-2018$} & 281 & & \\
\hline & 1553 & 2400 & \\
\hline $29-6-2018$ & & \multicolumn{2}{|c|}{ Friday } \\
\hline $30-6-2018$ & 227 & 300 No of Blocks & $24.33 \%$ less production than \\
\hline
\end{tabular}

3.1.2 Carpenter Gang Production: Carpenter gang was consisting of one charge hand, Five Carpenters and five helpers total ten numbers of operatives are involved in one gang.
According to gang and carpentry activities targets were already set by the company, there productivity was recorded on daily basis as shown in Table 3 .

Table 3. Production Record of Carpenter Gang

\begin{tabular}{|c|c|c|c|}
\hline Date & Production by Gang & Targeted Production & Remarks \\
\hline $1-6-2018$ & \multicolumn{3}{|c|}{ Friday } \\
\hline $2-6-2018$ & 41 & \multirow{6}{*}{$\begin{array}{c}48 \mathrm{~m}^{2} \text { is targeted production per day of } \\
1 \text { carpenter gang }\left(48 \times 6=288 \mathrm{~m}^{2}\right) \text { per } \\
\text { week }\end{array}$} & \multirow{6}{*}{$\begin{array}{l}\text { Shuttering Pad/ Foundation/tie } \\
\text { Beam (Fix \& Strike) } \\
16.667 \% \text { less production than the } \\
\text { targeted }\end{array}$} \\
\hline $3-6-2018$ & 39 & & \\
\hline 4-6-2018 & 43 & & \\
\hline $5-6-2018$ & 37 & & \\
\hline 6-6-2018 & 44 & & \\
\hline \multirow[t]{2}{*}{$7-6-2018$} & 36 & & \\
\hline & 240 & 288 & \\
\hline $8-6-2018$ & \multicolumn{3}{|c|}{ Friday } \\
\hline 9-6-2018 & 39 & \multirow{6}{*}{$\begin{array}{c}42 \mathrm{~m}^{2} \text { is targeted production per day of } \\
1 \text { carpenter gang }\left(42 \times 6=252 \mathrm{~m}^{2}\right) \text { per } \\
\text { week }\end{array}$} & \multirow{6}{*}{$\begin{array}{c}\text { Shuttering Column (Fix and Strike) } \\
12.301 \% \text { less production than the } \\
\text { targeted }\end{array}$} \\
\hline 10-6-2018 & 35 & & \\
\hline $11-6-2018$ & 33 & & \\
\hline 12-6-2018 & 41 & & \\
\hline 13-6-2018 & 38 & & \\
\hline \multirow[t]{2}{*}{$14-6-2018$} & 35 & & \\
\hline & 221 & 252 & \\
\hline
\end{tabular}




\begin{tabular}{|c|c|c|c|}
\hline 15-6-2018 & \multicolumn{3}{|c|}{ Friday } \\
\hline 16-6-2018 & 51 & \multirow{6}{*}{$\begin{array}{c}60 \mathrm{~m}^{2} \text { is targeted production per day of } \\
1 \text { carpenter gang }\left(60 \times 6=360 \mathrm{~m}^{2}\right) \text { per } \\
\text { week }\end{array}$} & \multirow{6}{*}{$\begin{array}{l}\text { Shuttering for Suspended slab (Fix } \\
\text { and Strike) } \\
21.112 \% \text { less production than the } \\
\text { targeted }\end{array}$} \\
\hline 17-6-2018 & 55 & & \\
\hline 18-6-2018 & 46 & & \\
\hline 19-6-2018 & 41 & & \\
\hline 20-6-2018 & 48 & & \\
\hline \multirow[t]{2}{*}{ 21-6-2018 } & 43 & & \\
\hline & 284 & 360 & \\
\hline $22-6-2018$ & \multicolumn{3}{|c|}{ Friday } \\
\hline 23-6-2018 & 43 & \multirow{6}{*}{$\begin{array}{c}54 \mathrm{~m}^{2} \text { is targeted production per day of } \\
1 \text { carpenter gang }\left(54 \times 7=378 \mathrm{~m}^{2}\right) \text { per } \\
\text { week }\end{array}$} & \multirow{6}{*}{$\begin{array}{c}\text { Shuttering for Parapets and } \\
\text { Upstands (Fix and Strike) } \\
16.931 \% \text { less production than the } \\
\text { targeted }\end{array}$} \\
\hline 24-6-2018 & 47 & & \\
\hline 25-6-2018 & 50 & & \\
\hline 26-6-2018 & 39 & & \\
\hline 27-6-2018 & 41 & & \\
\hline 28-6-2018 & 49 & & \\
\hline $29-6-2018$ & \multicolumn{3}{|c|}{ Friday } \\
\hline $30-6-2018$ & 45 & 54 & \\
\hline & 314 & 378 & \\
\hline
\end{tabular}

3.1.3 Steel Fixer Gang Production: Steel Fixer gang was also consisting of one charge hand, Five Steel Fixer and five helpers' total ten numbers of operatives are involved in one gang. According to gang and rebar activities targets were already set by the company, there productivity was recorded on daily basis as shown in Table 4 .

Table 4. Production Record of Steel Fixer Gang

\begin{tabular}{|c|c|c|c|}
\hline Date & Production by Gang & Targeted Production & Remarks \\
\hline $1-6-2018$ & \multicolumn{3}{|c|}{ Friday } \\
\hline $2-6-2018$ & 1750 & \multirow{6}{*}{$2100 \times 6 \mathrm{~kg}$} & \multirow{6}{*}{$\begin{array}{l}\text { Rebar Fixing to Pads and tie beams } \\
12.087 \% \text { less production than the targeted }\end{array}$} \\
\hline $3-6-2018$ & 1820 & & \\
\hline 4-6-2018 & 1922 & & \\
\hline $5-6-2018$ & 1782 & & \\
\hline $6-6-2018$ & 1875 & & \\
\hline \multirow[t]{2}{*}{$7-6-2018$} & 1928 & & \\
\hline & $11077 \mathrm{~kg}$ & $12600 \mathrm{~kg}$ & \\
\hline $8-6-2018$ & \multicolumn{3}{|c|}{ Friday } \\
\hline $9-6-2018$ & 1532 & \multirow{6}{*}{$1800 \times 6 \mathrm{~kg}$} & \multirow{6}{*}{$\begin{array}{l}\text { Rebar fixing to columns and beams } \\
11.120 \% \text { less production than the targeted }\end{array}$} \\
\hline $10-6-2018$ & 1720 & & \\
\hline 11-6-2018 & 1685 & & \\
\hline $12-6-2018$ & 1525 & & \\
\hline $13-6-2018$ & 1652 & & \\
\hline \multirow[t]{2}{*}{$14-6-2018$} & 1485 & & \\
\hline & 9599 kg & $10800 \mathrm{~kg}$ & \\
\hline $15-6-2018$ & \multicolumn{3}{|c|}{ Friday } \\
\hline $16-6-2018$ & 2100 & \multirow{6}{*}{$2400 \times 6 \mathrm{~kg}$} & \\
\hline $17-6-2018$ & 2112 & & \\
\hline $18-6-2018$ & 2052 & & Rebar fixing to raft foundation \\
\hline $19-6-2018$ & 2175 & & $10.819 \%$ less production than the targeted \\
\hline 20-6-2018 & 2225 & & \\
\hline \multirow[t]{2}{*}{$21-6-2018$} & 2178 & & \\
\hline & $12842 \mathrm{~kg}$ & $14400 \mathrm{~kg}$ & \\
\hline $22-6-2018$ & \multicolumn{3}{|c|}{ Friday } \\
\hline $23-6-2018$ & 1275 & \multirow{3}{*}{$1500 \times 7 \mathrm{~kg}$} & \multirow{3}{*}{$\begin{array}{l}\text { Floor slabs } \\
14.733 \% \text { less production than the targeted }\end{array}$} \\
\hline 24-6-2018 & 1303 & & \\
\hline $25-6-2018$ & 1282 & & \\
\hline 26-6-2018 & 1325 & & \\
\hline $27-6-2018$ & 1185 & & \\
\hline $28-6-2018$ & 1325 & & \\
\hline $29-6-2018$ & \multicolumn{3}{|c|}{ Friday } \\
\hline $30-6-2018$ & 1258 & & \\
\hline & $8953 \mathrm{~kg}$ & $10500 \mathrm{~kg}$ & \\
\hline
\end{tabular}




\section{ANALYSIS}

Production of every activity was analyzed based on gangs involved.

4.1 Mason Gang Production Analysis: Based on the targeted masonry activities, productivity of masonry work achieved seems to be keep on deceasing with respect to increase in temperature as shown in Figureure 1. In every week there was a certain decrease in the productivity of mason gang. In first week, it was recorded as $26.7 \%$ decrease, $2^{\text {nd }}$ week it was recorded as $22.96 \%, 3^{\text {rd }}$ week it was recoded as $30.088 \%$ \& in last week of month it was $35.29 \%$ recorded less production. The average monthly loss of mason gang production was $28.759 \%$. This continuous decrease in production of masonry work can lead to extensive delays in completing construction project. There was not a single week, where the mason gang has met their target.

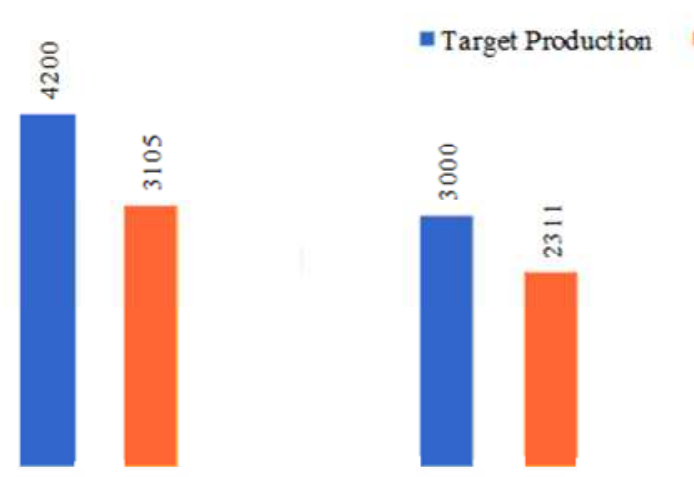

WEEK 1 : HOLLOW BLOCK WEEK 2: SOL ID BLOCK (1 0 CM THICK) (10 CM THICK)

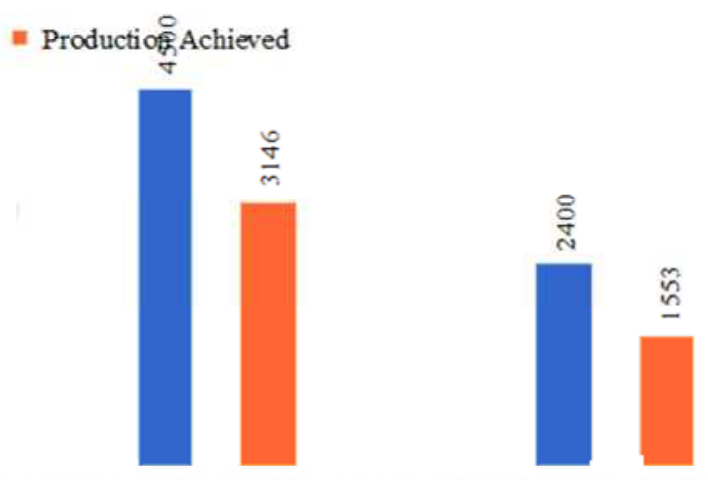

WEEK 3: HOLLOW BLOCK WEEK 4: SOL ID BL OCK

Figureure 1. Masonry Production Analysis

4.2. Carpenter Gang Production Analysis: The production of carpentry gang was also found with descending order. But the productivity of carpentry gangs seems to be more improved as compared to masonry gang. However, there was also loss of production from carpentry gang side with average monthly rate of $16.74 \%$.

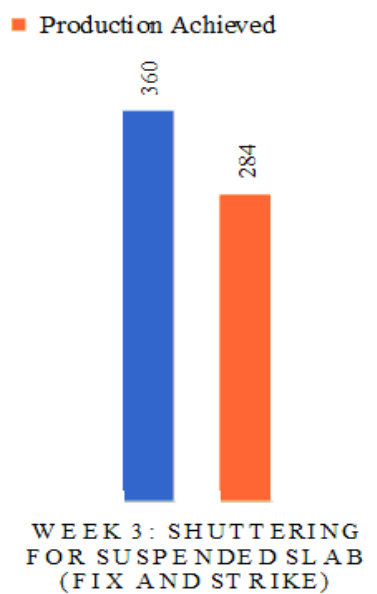

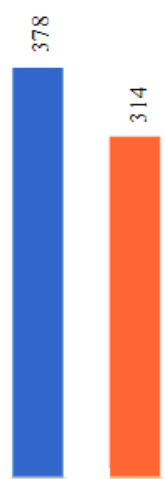

WEEK 4: SHUT TERING FOR P AR APETS AND UPST ANDS (FIX AND
WEEK 1 : SHUTTERING BEAM (FIX \& ST RIKE))
- Target Production

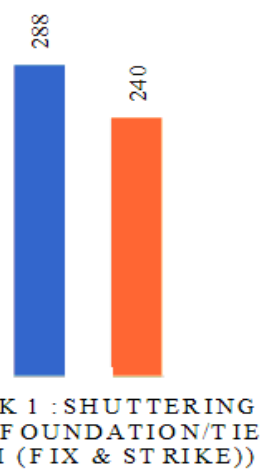

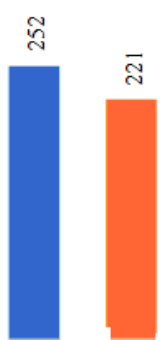

EEK 2: SHUT TERING STRIKE)
( 20 CM THICK)

Figureure 2. Carpentry Production Analysis

4.3. Steel Fixer Gang Production Analysis: The production of Steel fixer gang was much improved as compared to other gangs as shown in Figure 3. It is found from the results that
Steel fixer gang has tried their maximum efforts to meet the targets of company. However, there is also less production with the average monthly rate of $12.188 \%$. 


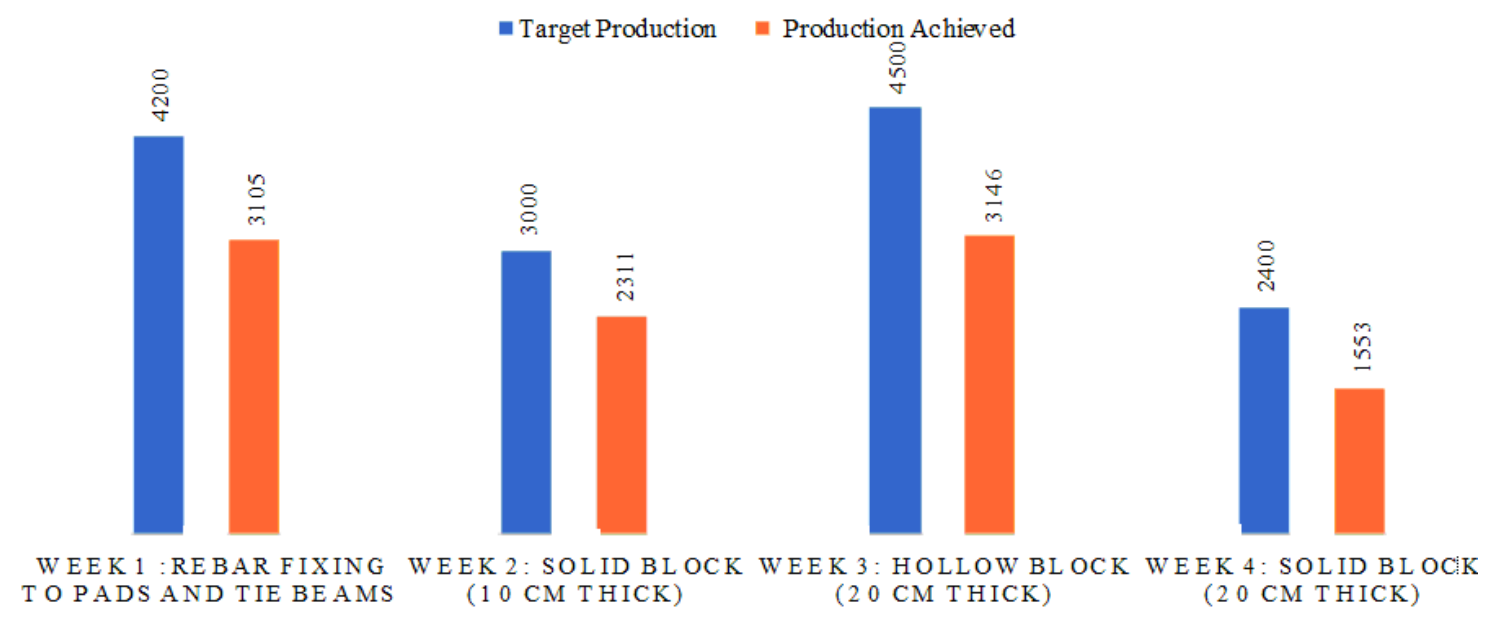

Figureure 3. Steel Fitter Gang Production Analysis

\section{CONCLUSION}

This study is focused on the arid climate environment. This study concludes that, prior to signing the contract for construction project. The location, environment, topography of region, capacity of construction operatives must be kept in mind to decide the proper timeline for the success of project. It is analyzed above that as the temperature was increasing the productivity of operatives was keep on decreasing. Besides the productivity, environment was also harshly affecting the health of construction operatives.

\section{References:}

I.A Bhatti et al. (2018). "Delay in High-rise Building Construction Projects of Dubai: A Review". Engineering Science and Technology International Research Journal, Vol.2, No.2, 2018, 7-15.

Buchan R. D., Fleming F. W., Kelly J. R. (1993). Estimating for builders and quantity surveyors, Oxford: Butter worthHeinemann.

I.A Bhatti et al. (2018). "Replacement of In-situ Bathrooms with POD Bathrooms to Save Time \& Money within construction of fast track projects (Project Case study)". Asian Journal of Technical Vocational Education and Training (AJTVET). Vol. 5, No.9, 2018, 42-45.

Oladiran, O. J. and Onatayo, D. (2019). "Labour productivity: Perception of site managers on building projects" LAUTECH Journal of Civil and Environmental Studies Vol.2, No.1, 2019, $1-10$.

Jarkas, A. M. \& Bitar, C. G. (2012). Factors affecting construction labour productivity in Kuwait. Journal of Construction Engineering and Management 138(July): 811820.

Revianty Nurmeyliandari Nurhendi, Muhamad Azry Khoiry, Noraini Hamzah (2019). "Review on Factors Influencing Labour Productivity in Construction Project" International
Journal of Recent Technology and Engineering (IJRTE) ISSN: 2277-3878, Vol.7, No.6.

S. Sohu et al. (2018). "Significant Mitigation Measures for Critical Factors of Cost Overrun in Highway Projects of Pakistan" Engineering, Technology \& Applied Science Research Vol. 8, No. 2, 2018, 2770-2774.

I.A Bhatti et al. (2018). "Implementation of Building Information Modeling (BIM) in Pakistan Construction Industry" Engineering, Technology \& Applied Science Research Vol. 8, No. 4, 2018, 3199-3202.

Kazaz, A., Ulubeyli, S., Acikara, T. \& Er, B. (2016). Factors Affecting Labour Productivity: Perspectives of Craft Workers. Procedia Engineering, hlm. $28 \quad-\quad 34$. doi:10.1016/j.proeng.2016.11.588.

Mahamid, I. (2013). Contractors' perspective toward factors affecting Labour productivity in building construction. Engineering, Construction and Architectural Management 20(5): 446-460. Doi: 10.1108/ECAM-08-2011-0074.

Gerek, İ. H., Erdis, E., Mistikoglu, G. \& Usmen, M. (2015). Modelling masonry crew productivity using two artificial neural network techniques. Journal of Civil Engineering and Management 21(2): 231-238. doi:10.3846/13923730.2013.802741.

Song, L. \& Abourezk's, S. M. (2008). Measuring and Modeling Labour Productivity Using Historical Data. Journal of Construction Engineering and Management 134(10): 786-794. Doi: 10.1061/ (ASCE) 0733- 9364(2008)134:10(786). 